\title{
Concepts for an Annular Pole Piece Detector for the Simultaneous Measurement of $X$-Rays and Backscattered Electrons Inside a SEM
}

\author{
A. Liebel ${ }^{1}$, R. Eckhardt ${ }^{1}$, M. Bornschlegl ${ }^{1}$, A. Bechteler ${ }^{1}$, A. Niculae ${ }^{1}$, H. Soltau $^{1}$ \\ ${ }^{1}$ PNDetector GmbH, Emil-Nolde-Str.10, D-81735 München, Germany
}

In modern Scanning Electron Microscopes (SEM) a large variety of different signals is collected to create images with various contrasts or to receive compositional information. Among others, Backscattered Electrons (BSE) and characteristic X-rays belong to the major signals which are collected routinely. Both give information about the sample composition and therefore complement each other very good as exemplarily shown in Figure 1. Since electrons and photons follow a straight path out of the sample the collection efficiency is weak if the detectors are far away from the sample resulting in a small solid angle in which they are collected. Hence, in order to collect them with high efficiency modern BSE and Energy Dispersive X-Ray (EDX) detectors use large active areas and are positioned close to the sample.

A possibility to detect both, BSE and X-Ray signals, simultaneously with high efficiency has been proposed already in 2009 [1]. A promising way for achieving this is to use annular detectors which are positioned right underneath the pole piece and therefore very close to the sample. Recently, measurements with the annular pole piece EDX detector "Rococo2" have been presented by us [2]. Figure 2 shows the detector assembly and the filter foil configuration which was used for stopping the backscattered electrons. The transmission of the used combination of thin Beryllium and Mylar foils resulted in a continuous sensitivity for X-Rays over a wide energy range down to the carbon line. The detector has four kidney shaped Silicon Drift Detector (SDD) cells which results in a huge solid angle of up to $1.4 \mathrm{sr}$. This is about 100 times larger than with a conventional SDD with $10 \mathrm{~mm}^{2}$ active area. BSE detectors with optimized geometry which are positioned close above the sample can collect up to about 60 percent of the electrons backscattered from the sample which highly increases the contrast and signal to noise ratio of the BSE image [3]. However, by using standard individual EDX and BSE detectors at the conventional positions, both signals cannot be acquired with such a high efficiency at the same time which leads to the idea of using a combined annular detector for backscattered electrons and X-Rays.

Figure 3 shows two different concepts for such a detector with the BSE detector positioned either above the annular EDX detector or with both detectors at the same level. Compared to the existing Rococo2 EDX detector the central hole is enlarged which slightly decreases the solid angle but provides the possibility to implement the simultaneous detection of backscattered electrons at high take off angles.

We will present and evaluate calculations of the solid angle and the collection efficiency of different combinations of annular EDX and BSE detectors and compare the resulting images or spectra. The pros and cons of such a combined detection system will be discussed.

[1] H.Soltau et al., Microscopy \& Microanalysis, vol. 15, S2 (2009), pp. 204-205

[2] A.Liebel et al., Microscopy \& Microanalysis, vol. 19, S2 (2013), pp. 1134-1135

[3] A.Liebel et al., Microscopy \& Microanalysis, vol. 18, S2 (2012), pp. 1206-1207 


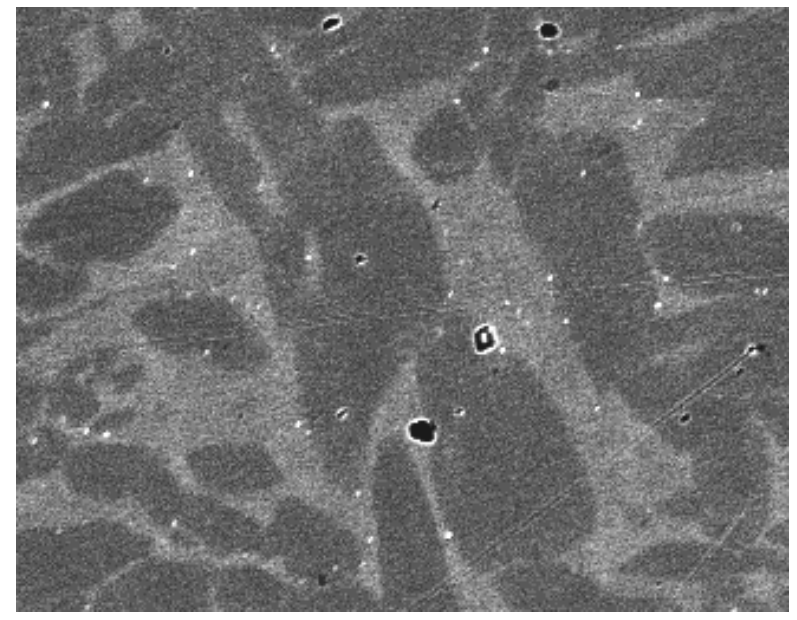

a)

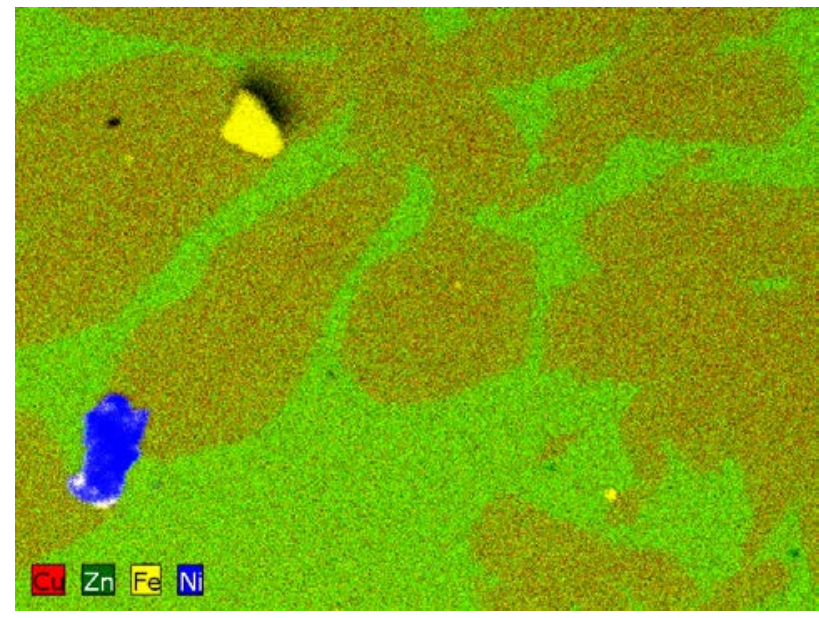

b)

Fig. 1. a) BSE image of a duplex brass sample showing $\alpha$ and $\beta$ phases with different concentration of copper and zinc and b) an EDX mapping of a similar sample acquired with the Rococo2 SDD detector.

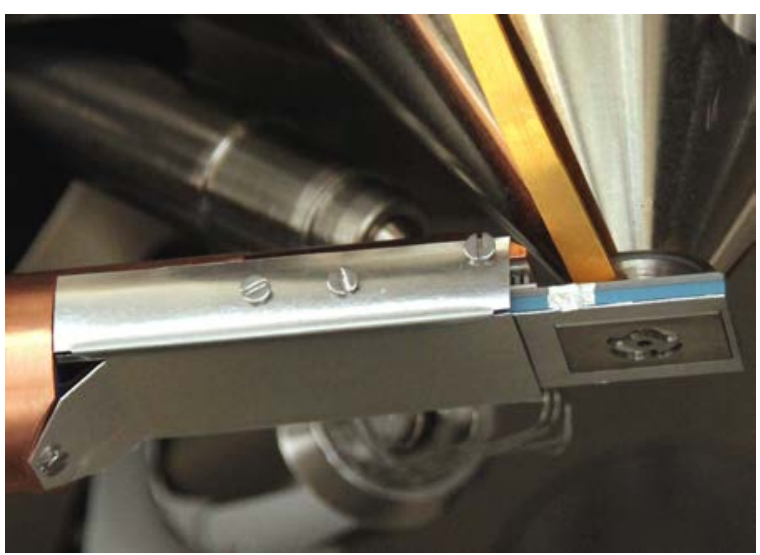

a)

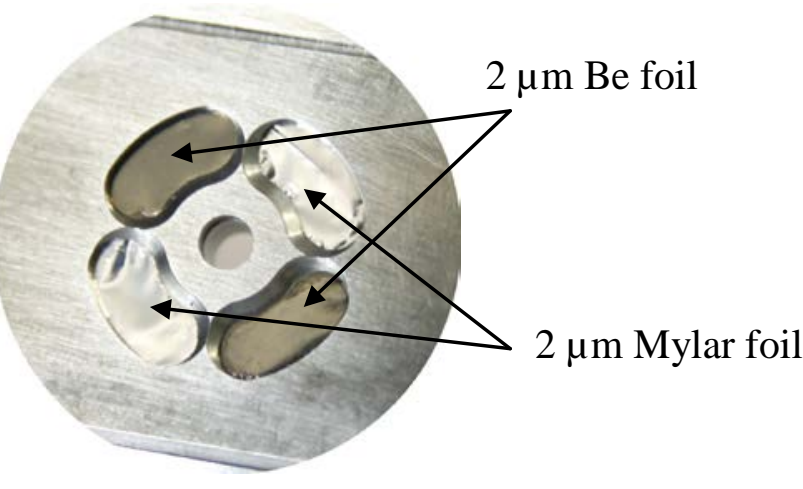

b)

Fig.2. a) The annular pole piece EDX detector "Rococo2" inside the SEM and b) a view at the top of the detector showing the collimator with a filter combination of $2 \mu \mathrm{m}$ Be and $2 \mu \mathrm{m}$ Mylar.

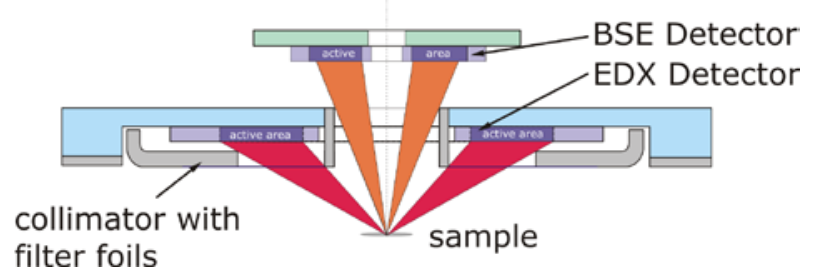

a)

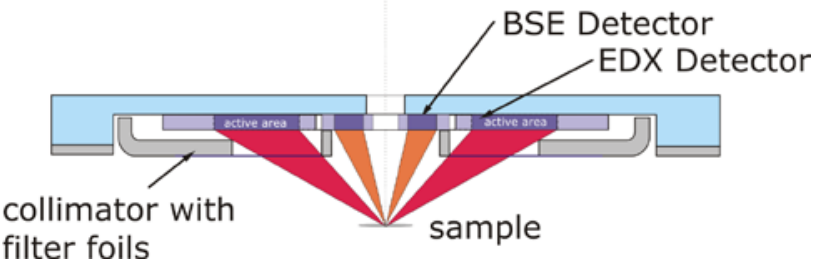

b)

Fig.3. Schematic drawings of a combined EDX and BSE detector setup with a) the BSE detector positioned above the annular EDX detector and b) the two different detectors positioned on the same level. 